\title{
XIV.
}

\section{Der königliche Leibarzt und Hofastrologe Johann Thibault, Michael Servet's Pariser Freund.}

\author{
Von H. Tollin, \\ Lic. theol, und Prediger zu Magdeburg.
}

Motto: Experientia rerum magistra.

Ein Geist, der seinem Jahrhundert voraufeilt, wird selten Freunde haben, denn den Scheiterhaufen theilt so leicht kein Freund.

Auch Michael Servet, der spanische Polyhistor, der in der Theologie, Philosophie, Jurisprudenz, Geographie, Medicin und Physiologie als Reformator aufgetreten ist, hat bei seinen Lebzeiten wenige Freunde gezäblt. Gesinnungsgenossen fand er in Augsburg, Basel, Strassburg, Hagenau, Frankfurt a. M., Erfurt, Vlm und Speier; in Bologna, Padua, Venedig, Vicenza, Siena und Neapel; in Toulouse, Lyon, Charlien, Poitiers, Paris, Vienne und Genf; in der Gefahr aber stand ihm selten Jemand zur Seite.

Um so mehr ist es Pflicht des Geschichtsschreibers, solehen Beistand in Gefahr vollauf zu würdigen. Darf doch auch in der Geschichte der Medicin der Forscher seine Gestalten nicht zu blossen Namen verschrumpfen lassen, noch ihr Leben zu blossen BücherCatalogen. Es müssen lebendige Menschen sein, mit denen man zu thun hat, Wesen von Fleisch und Blut, Charaktere mit bestimmten Neigungen und Anlagen.

Michael Servet hat drei Prozesse gehabt.

In dem Genfer Prozess, der mit seinem Scheiterhaufen abscbloss, stand ihm Noble Amy Perrin zur Seite, bis Servet's Sache völlig verloren war: dann trennte er sich und erhielt sich dem Vaterland.

In dem Vienner Prozess stand ihm Balthasar Arnoullet zur Seite. Sie mussten beide fliehen und ihr Bildniss wurde gemeinsam verbrannt. 
In dem Pariser Prozess gegen die medicinische Facultät stand dem Servet der Leibarzt König Franz I. von Frankreich, Johann Thibault zur Seite.

Jehan Thibault war seiner Zeit ein bei Hoch und Niedrig ebenso innig geliebter und verehrter, wie energisch gehasster Mann. Er bat. in der Pest tausenden von armen Lenten beigestanden, vielbegehrte Bücher geschrieben, einen sehr bedeutenden Einfluss auf seine Umgebung geübt. Dennoch wird weder bei Kurt Sprengel noch bei Haeser noch bei Baas sein gedacht. Ja selbst von den Servet-Biographen widmet nur Mosheim ${ }^{1}$ ) inm eine Anmerkung.

Jehan Thibault war der erste protestantisch gerichtete Niederländer ${ }^{2}$ ), den wir mil dem spanischen Antitrinitarier in officieller Verbindung finden und von dem vielleicht der antitrinitarische Einfluss ausging, den Michael Servet später auf gewisse niederländische Kreise übte.

Jehan Thibault war aber auch ein Practiker. Darin lag sein Erfolg begriffen und die Ursache seiner Verfolgung.

Im Prozess, den er selbst am 2. März 1535 mit der medicinischen Facultăt von Paris baite, bezengt er, dass er „auf mehreren berühmten Universitäten die freien Künste und die Arzneiwissenschaft studirt, aber dabei allerdings sich mehr um die empirische Medicin bekümmert habe, als um jene Logik, die Plinius Clinika nenne. Darauf habe er, als Leibarzt der seligen östreichischen Erzherzogin Margarethe von Flandern in Niederdeutschland, Holland, Seeland, Flandern und anderen Orten practisirt. Als nun aber der König Franz 1. in Cambray war, hörte er von Thibault's Wissen und Erfahrung (expérience), liess ihn zu sich bitten und schlug ihm vor, falls er sich nach Frankreich zurüekzöge, ihm so viel Gutes zo thun, wie ihm kein anderer Fürst thun könnte. Als nun Thibault auf dies Königswort hin die Stadt Nevers, wo er eine starke ärztliche Praxis und fünf oder sechs Kinder hatte, verlassen,

1) Anderweitiger Versuch einer vollständigen und unparteiischen Ketzergeschichte. Helmst. 1748. $4^{0}$. S. 76 Anm.

2) Auch ein anderer Niederländer des Namens Thibault erscheint als Astrologe: es ist Odoard Thibault, mathématicien, demeurant à Louvain en Flandres, l'an 1550. Zu Paris erschien von ihm une prognostication bei Guil. Niverd, pour l'an 1550; auch zu Rouen ein Almanach pour l'an 1551 bei Guill. de la Mothe. S. De la Croix du Maine: Art. Thibault. 
und auch sein Weib sich ihm angeschlossen batte, in Hoffinung auf das Versprechen des Königs, hätte er sich kaum in dieser Stadt paris befunden, als man ihm allerlei Schmach anzuthun unternahm, ihn gefangen setzte, endlich aber wieder aus dem Gefängniss entliess. Sobald er jedoch wieder practisiren wollte, sei er von Neuem durch die medicinische Facultät dieser Universităt daran verhindert worden und bier vor Gericht citirt $^{1}$ ).

Mit dieser gerichtlich beschworenen Selbstaussage Jean Thibault's stimmt, was wir sonst ron inm wissen.

Der berühnte rheinländische Polyhistor Henricus Cornelius Agrippa von Nettesheym, immer arm, ehrlich und seltsam, aber durch sein unermessliches Wissen und seine ausgedehnten Beziebungen für sich eine Welt, nimmt den ärztlichen Collegen in Schutz gegen die Zunft der Aerzte von Autwerpen. Der ehrenwerthe Magister (honorabilis vir magister) batte gegen die Verleumdungen jener ihn sich zur Hülfe gerufen, damit Agrippa, einst selber Arzt bei Königen und Fürsten (apud reges principesque), ihm, dem offentlichen Arzt und Astrologen (medicae et astrologiae professor), bezeugen sollte, dass er ein für seinen Beruf genügendes Wissen habe (de medicae artis sufficientia). Agrippa hält die dem Thibault gemachten Vorwürfe für ein grobes Unrecht (mera injuria), und sieht sich zu der Erklärung bewogen, dass, wenn in Antwerpen ihm oder den Seinen eine Krankheit zustösse, er sich lieber in Thibault's Hände begeben würde, als in die der ubrigen Aerzte. Komme es doch in der Medicin nicht darauf an, mit Logik und Sophistik zu glänzen, sondern kranke Leute gesund zu machen; und diese Medicin sei Sache der Praxis [mechanicam et operatricem medicinam $]^{2}$ ). Auch sei es offenkundig, dass Magister Theobald sehr viele (plurimos) von denen, welche die anderen Antwerpener Aerzte aufgegeben bätten, hergestellt habe durch seine Erfahrung. Auch seì das die Ursache des Neides, dass sie, die doch Doctoren

1) Do Boulay, Histor. Academ. Paris VI. 264 sq. (Extrait des Régîtres du Parlement de Paris): Gontra Doctorem Empiricum.

$\left.{ }^{2}\right)$ Aehnlich schilt Paracelsus auf die heillosen Lotterbuben, die, ihrem Eigennutz, Gewissenlosigkeit und Rohheit zu Liebe, vichts wüssten als ein bischen griechische Sprache, und meinten, weil die Sprache die Bücher regiert, regiere sie auch die liranken. Aber gute Erasmianer seien noch keine guten Aerzte (III, 160). - Vgl, Lessing, Leben des Paracelsus, 29. 
sind, besiegt wïrden durch Idioten (quia ipsi doctores cum sint vincuntur ab idiotis). Als jene Schwitzpestilenz (sudoriferum illud malum) uber diese Stadt hereinbrach, haben da nicht die Stadtärzte, gegen ihren dem Magistrat geleisteten Eid und obwohl sie für ihre Dienste öffentlich bezahlt werden, ibre Patienten verlassen? Und während Magister Jean Thibault und wenige Andere mit Anstrengung und Nutzen Hulfe bringen, steht es jenen scholastischen Aerzten (istis scholasticis medicis) und ellenlangen Roben-Doctoren (sesquipedalibus doctoribus) frei, ihre Sophismen aneinander zu reihen und über unser Leben und Gesundheit mit Hornvieh-Schlüssen hin und her zu streiten und in dunklen Sentenzen Urtheil zu sprechen ${ }^{1}$ ).

Diese Eingabe des Cornelius Agrippa, des damaligen kaiserlichen Archivraths (Caesareae majestatis a Consiliis et archivis Indiciarius) in Mecheln, der vom 23. Juli 1528 bis Ende 1530 in Antwerpen gelebt hatte, scheint beim kaiserlichen Parlament (senatus) von Mecheln, angesichts der Pesterfolge Thibault's, für das Jahr 1530, aus dem sie datirt, zar Niederschlagung der Klage genügt zu haben.

Auch giebt Thibault im folgenden Jahre eben zu Antwerpen seinen Trésol du remède préservatif heraus ${ }^{2}$ ). Das Buch ist selten geworden. Ob ausser dem Pariser Exemplar (Biblioth. nationale T. $1045 \mathrm{~B}$ ), das ich 1859 benutzen durfte, ein zweites existirt, wage ich nicht zu entscheiden. Das Buch wird gemeinhin als Thesaurus Pestis citirt, obwohl es auch noch viele andere Krankheiten behandelt, wie schon der Titel zeigt ${ }^{3}$ ). Thibault nennt sich da der kaiserlichen Majestät Arzt und Astrologe. Er widmet die Schrift dem sehr tugendhaften, berühmten, sehr gelehrten und edlen Herrn

1) Agrippa, Opp. apud Beringos fratres. II, Ep. 7 Lib. V1. a. 1530.

2) Imprimé en Anvers par Martin Lempereur. L'an MDGXXXI. Die Schrift bat nor 24 Seiten.

3) Le thresor du remede preservatif et guerison (bien experimentee) de la Peste et fievre pestilentiale; avec declaration dont procedent les gouttes naturelles, et comme elles doibvent retourner. Et aussy aucunes allegations et receptes sur le mal caduque. Pleuresies et Apoplexies et cequil appartient a ung parfaict medecin etc. Compose par Maistre Jehan Thibault medecin et astrologue de limperiale maieste etc. Das Buch fübrt deutsche Lettern (gothicam formám). Erst Simon Colinaeus führte in Frankreich die Italicos characteres ein (Maittaire: Annales typogr. II, 403). 
Hieronymus van der Noot, Kanzler von Brabant, dem Haupt, Liebhaber, Grundpfeiler und Vertheidiger aller Derer, welche Wissen baben und Wissenschaft lieben. Die Schrift trägt das Motto: Experientia rerum magistra; darüber des Buchbändlers marktschreierische Empfehlung ${ }^{1}$ ).

Da Thibault 1531 Kaiser Garl V. Arzt und Astrologe ist, braucht er mit seinem Wissen über den bevorstehenden Lauf der Gestirne nicht zurückzubalten. Auch beginnt er die Widmung, „er habe den Einfluss des Himmelslaufs und die Lebensverfassung und Lebensweise der gegenwärtigen Welt (complexion et maniere de vivre du monde a present) in Erwägung gezogen und daraus erseben, dass uns noch mehrere verschiedene Krankbeiten bevorständen, insbesondere Pesten, Apoplexien, langwierige Fieber, plötzliche Tode, Pleuresien u. a., die den der Astrologie unkundigen Aerzten fremd bleiben. $\mathrm{Er}$ babe deshalb auch mit seinen auf $\mathrm{Er}-$ fahrung begründeten Vorbeugungs- und Heilmitteln nicht zurückhalten wollen. Und da nun eine Anzahl ehrenwerther Leute, so Herren als Damen verwirrt worden sind und in Sorgen, nach welchen Grundsätzen man sich den Arzt zu wählen habe, so füge er einige Punkte hinzu, worauf es bei einem wahren und vollkommenen Arzte ankommt. Sage doch schon Sokrates, der Mensch wird gebessert durch Erfahrung (l'homme est corrigé par expérience), und Plato: der gute Rath wird gegeben durch viele und grosse Erfahrung (le bon conseil est donné par moult grande expérience). Und wenn hinwiederum Sokrates behauptet, der höchste Gewinn sei der eines ehrlichen Freundes, so möchte wohl kein geringerer Gewinn die Bewahrung eines solchen sein. Un nun diese mir angelegen sein zu lassen, kann ich nicht die viejen von Dir empfangenen Wohlthaten, wie Plato räth, vergelten - denn ich besizze nicht dieser Welt Gäter. Darum biete jch Dir, der der armen Waisen Zuflucht und Tröster ist, meine schwache Kenntniss, geringes Verständniss und schuldigen Dienste an in dieser kleinen Schrift. Mag sie allen Ehrenleuten helfen, hauptsächlich auch jenen vielen Armen

1) Au lecteur:

Ti ne me peulx trop acheter

Ne trop garder ny estimer

Sy tu veulx en moy proufiter

Lis moy donc sans $y$ rien laisser. 
und andern, die nicht im Stande sind, die Meister und Apotheker zu bezablen"... 1).

In der Schrift selbst fragt sich Thibault zunächst, woher die befremdliche Thatsache käme, dass bei jeder seltsamen Krankheit die höchstbetitelten und höchstberufensten Aerzte am wenigsten Erfabrung (le moins d'expérience) und Kenntniss bewiesen? Das kommt, antwortet Thibault, aus keinem anderen Grunde, als weil sie nichts verstehen von der edlen Kunst und Wissenschaft der Astrologie, durch die man erst im Stande ist, die Complexion des Kranken, die Disposition seiner Krankheit, die Zeit der Hejlung oder seines Todes vorber zu erkennen, wie uns Haly (Abbas), Ptolemaeus, Alchabitius und Johannes de Sanonia (Saxonia?) unter dem Texte des Alchabitius lehren. Und Hippokrates sagt im Abschnitt über die Veränderung der Luft, die Astrologie ist nicht blos ein kleiner Theil der Medicin, sondern sie ist die ganze. Wie will man auch zukünftige Krankheiten vorhersagen, als durch Astrologie oder eine besondere göttliche Gnade (ou par grace divine)? Was aber die heutigen Theoretiker von der Praxis verstehen, das kann man in zwei Monat lernen, sowobl betreffs der Urinaussagen als zur Verschreibung von fecepten und wie man den Puls fassen muss? Findet man das doch alles schon niedergeschrieben in Büchern. Auch ist diese Kenntniss nicht in's Volk gekommen durch die Gelehrten oder die Grosstitelträger, sondern durch die Einfältjgen, denen Gott die Gnade gegeben hat, die Wahrheit zu erkennen. Und noch heute, wenn Gott der Welt das Verständniss oder das Heilmittel für irgend eine unbekannte Krankheit geben wollte, so würde er die Erfahrung (l'experience) und Erprobung dieser Kenntniss doch immer den Einfältigen (simples) zur Verbreitung und Offenbarung überlassen. Und unter allen Gnaden von Kenntnissen (entre toutes les graces des sciences) ist die edelste doch die Kunst und Kenntniss von der Astrologie, welche unser Herr (nostre seigneur) hauptsächlich den Armen und Einfältígen überlässt. Denn offenbar haben wir aus uns selber keine Macht, irgend eine Kenntniss zu erwerben und darin ein guter Arbeiter zu sein, es sei denn dass unserer Natur dazu das besondere Gnadengeschenk verliehen

1) et principalement a plusieurs poures et autres lesquelz nont point pour payer les maistres ni appotiquaires. 
wird, wie ich das in meiner Antwort gegen Meister Gaspar Laet aus dem Ptolemaeus gezeigt habe ${ }^{3}$ ). Hat man doch mehrere grosse Gottesgelehrte gefunden (plusieurs grands clercs en theologie), die nicht im Stande waren, die Kunst der Astrologie zu verstehen. Geradeso verhält es sich mit allen anderen Wissenschaften, deren Verstänđ̊niss schwer fällt, falls man nicht zu ihrer Natur götllich berufen worden isl. Der gute Wille rejcht da nicht aus. Und wenn dann solche Unberufenen es durch ihren Willen ertrotzen wollen, so entstehen nur Schrecken, Missbrauch und grobe Fehler, und werden solche ihre Kranken in die Grube mediciniren (les medicineront en la fosse) ${ }^{2}$ ). Denn sie vertrauen auf ihre Schriftgelehrsamkeit (clergie) und auf die Kunstausdrücke ihrer Wissenschaft, ohne noch zu wissen, wann man Medicin reichen oder wann damit aufhören muss? Sehr wahr sagt darum Franciscus Petrarca, dass man sich vor einem gelehrten Arzt wohl hüten muss, weil er sich mehr auf sein Wissen verlässt, als auf die Disposition und Veränderung der Krankheit seines Patienten."

„Um nun dem Missbrauch zu steuern, beginnt Thibault damit, den Medicinern und Medicinerinnen (medecineresses) zu erklären, was sie wissen und kennen müssen. Er führt die 10 Stücke auf, die seit Ptolemaeus, Haly-Abbas und Alkindi traditionell geworden waren ${ }^{3}$ ). Der siebente Punkt betrifft die Zeit der guten und der bösen Krisis. Man müsse dabei auch beobachten, wie an den kritischen Tagen der Mond steht und ob er mit guten oder bösen Planeten sich vereinige und ob der Anblick des Himmels gut oder böse sei? Wer aber leugnet, dass astrologische Kenntniss für den Mediciner unumgänglich sei, der mag kühnlich wider mich schreiben (quil escripve hardiment contre moy). Dann erläutert Thibault die Ursachen, warum man sich bisher in der Kur der Pest geirrt

1) Diese Responce contre maistre Gaspar Laet scheint gedruckt worden zu seio.

2) Maintenant nous faisons apprendre à nos enfans ce que bon nous semble. Et ce est la cause que plusieurs sont destruictz, et viennent à perdre tout ce que on leur met entre les mains. Et après quilz sont privez de tous leurs biens, ehors viennent à faire aultre practique ou mestier tel que nature leur ensejgne, et dont ilz sont enclins... Nature delle mesme ramaine son homme là où il doibt estre (p, 5). Gewjss ein beherzigenswerther pädagogischer Grundsatz!

$\left.{ }^{3}\right)$ Der 10 ist derselbe wie der 6 . 
hat, nehmlich deswegen, weil man ibre Ursache nicht kannte, und man müsse doch immer zuerst die Ursache kennen, ehe man das entscheidende Heilmittel recht wählen könne. Und was ist nun die Ursache der Pest? Es giebt deren fünf: Gewalt, Weiber, Hunger, Kälte und Schrecken ${ }^{1}$ ). Darauf werden die Merkzeichen avgegeben, woran man die wahre Pest erkennt, die beiden Gründe, die man wissen müsse, um diese Krankheit zu heilen; welche Ader man öffnen müsse um Kopf und Gedächtniss zu schützen? - Er räth, die feine Vene zu öffnen, die wir über den Augenlidern haben und die von den Augen niedersteigt über und in die Nase: diese Vene sei der Schlüssel des Leibes (laquelle est la clef du corps). Dagegen solle man sich wobl hüten, die Vena cardiaca oder basilica zu öffnen, so sehr auch die Alten dazu rathen mögen. Denn ich sage euch, wenn Avicenna, Herr Galenus und die Anderen beute lebten, so würden sie gerade so neu sein, wie die Aerzte, die man heute finden könnte. Denn die Zeit für ihre Schriften ist vorüber. Die Welt ist nicht mehr so, wie sie war zu jener Zeit: das liegt ja deutlich zu Tage ${ }^{2}$ ). Nichts bleibt verborgen ausser für den Undankbaren und für den Unwissenden (rien n'est absconse que pour l'ingrat et l'ignorant). Es ist deshalb das einfachste Ding der Welt, die Pest und das pestilentialische Fieber zu heilen ${ }^{3}$ ). Wie purgirt man nun, kommt die Pest von Kälte her? Da werden neun verschiedene Weisen angegeben, je nach den verschiedenen Umständen. Wie behandelt man aber den Pestkranken, sobald die Kraft der Krankheit gebrochen ¡st? Es werden Kräuter und Fleischarten, die gutes Blut erzeugen, angegeben; auch andere Kräuter zu anderen Zwecken. Folgt ein Präservativ sowohl für die Inficirten als für die anderen in Betreff der Pest, ein zweites, ein drittes; dann eine Conserve Morgens zu nehmen gegen alle Pestluft, um Herz und Magen zu kräftigen, dabei auch etwas abzuführen; jetzt aber das Recept zum Thibault'-

1) engendrée par cinq manieres toutes commencent par f, ascavoir force, femme, faim, froit et frayeur.

2) Je vous dis que sy Avicenne, Messire Galenus et autres estoient apresent an monde, quilz serolent aussy nouveaux que ceulx que on pourroit trouver: car le temps est passe de leurs escriptz, le monde nest pas tel quil estoit in fllo tempore, comme nous le voyons evidamment.

3) La peste, c'est la plus simple chose qui soit au monde pour guerir. 
schen Pestpulver; darauf eine (besondere) Abhandlung über Fallsucht, Apoplexie u. s. w.; die Kur der Apoplektischen; auch ein Syrop, der da vorbengt und heilt und die Katarrhe aus dem Gehirn entfernt, Morgens zu nehmen, eine Unze, wenn man will. $\mathrm{Zu}-$ letzt spricht er von den verschiedenen Gichtarten und wie sie alle zu vertreiben sind? Zum Schluss lobt Thibault den Namen unseres Herrn (le nom de nostre Seigneur), der ihm die Gnade gegeben hat, die Schrift zu Ende zu bringen; bittet aber auch die, welche ein Verständ̊niss für die ärztliche Wissenschaft haben, ihm seine rohe und einfältige Darstellung verzeihen $\mathrm{zu}$ wollen (ma rude et simple composition), da er ja noch nichts sei als ein armer Student (moy qui suis ung poure estudiant), der in der Wissenschaft erst zukünftig etwas leisten möchte, wozu Gott durch seine Gnade Wachsthum geben mag. Amen."

Man siebt, dass Thibault, im Jahre 1531, wenn auch kaiserlicher Leibarzt, auf den medicinischen Doctortitel keinen Anspruch macht. Er hat in der Pest Erfahrung gesammelt und freut sicb, seine Erfahrung mitzutheilen. Aber er bleibt Forscher. Der Mangel des Doctortitels musste aber den niederländischen Practikanten, wohin er sich auch wenden mochte, mit allen Facultäten der Welt in Collision bringen.

Sein zweiter Fehler war nun die Astrologie. Vom gemeinen Volk geachtet, von manchen Klugen benutzt, an den Höfen zum Theil wohl gelitten, war die Astrologie gerichtlich seit lange bei Todesstrafe verpönt.

Thibault's dritter Fehler war der Kampf gegen die eigene medicinische Schule. Mochte man den Arabern huldigen oder den Griechen, die geschulten Aerzte waren alle Dogmatisten. Wer zugleich Avicenna und Galen bekämpfte, der trat zwischen zwei Fever und weihte sich dem Untergang.

Dieser dreifachen Sünde schuldig kam Jean Thibault nach Paris. Es muss nicht gar lange nach der Herausgabe des Trésor de la peste gewesen sein. Denn schon unter dem ersten und zweiten Decanat des Johann Vassetz (Ende 1532 und Anfang 1533) finden wir, dass die Pariser medicinische Facultät Ausgaben um Jean Thibault's willen hat (Impensa facultatis medicinae pro necessariis facultatis et litizationibus) ${ }^{1}$ ). Energischer wird die Sache

1) Commentarii facultatis medicinae Parisiensis, die mir 1858-1859 die dortige 
betrieben, seitdem der in der Chirurgie als begabter Schüler des Guy von Chauliac berühmte Johann Tagault aus Amiens, ein höchst merkwürdiger Mann, auf den wir (anderswo) zurückkommen müssen, Decan wurde. Dieser trug Sorge, dass am 8. Mai 1534 eine Commission niedergesetzt wurde behufs Prüfung des unrechtmässig practisirenden Jean Thibault. Allerdings war er ja berufener und besoldeter Leibarzt des Königs, aber nach den 1436 und öfter ${ }^{1}$ ) bestätigten Statuten der Facultät hatte Niemand ein Recht, in Paris zu practisiren, der nicht unter Vorsitz des Prévost de Paris durch bewährte und von der Facultät berufene Aerzte geprüft worden wäre (vocatis medicis peritis) ${ }^{2}$ ). Die zur Prüfung Thibault's ausgewählten vier Aerzte hiessen Joh. Durnel, Ludv. Braillon, Michael Dumoncea und Johann Morelli (ad examinandum Jo. Theobaldum illicite practicantem). Braillon war ebenfalls Leibarzt des Königs.

Der verwegene Fremdling aber (adventicus practicans et audaculus), statt sich der Commission zu stellen ${ }^{3}$ ), vermehrte seine Schuld durch Herausgabe von Kalendern und jährijchen Zukunftsbüchlein (Almanachs et prognostications), die zwar dem König angenehm und dem Volke unentbehrlich, der Facultät aber äusserst verhasst waren, weil sie sich ihrer Censur entzogen hatten. Tagault, der Decan, hält darum den Leibarzt des Königs für einen ausgemachten Betrüger (impostorem maximum), und benutzt das nächste Promotions-Frühstück, um die Freude der Facultät ${ }^{4}$ ) zu erhöhen

medicinische Facultăt mit grösster Liebenswürdigkeit zur Durchforschung an Ort und Stelle erschlossen hat, wofür ich ibr jetzt noch Dank weiss.

1) z. B. 28. Januar 1512. S. du Boulay VI. p. $51 \mathrm{sq.}$

2) du Boulay VI. p. 33. - Sehr interessant ist der Prozess der Pariser Facultăt contra doctores extraneos 1506. In Montpellier promovirte man billiger. In Paris bostete 10 escus der Titel des bachelier, 20 escus der des licencié, $500-600$ escus der des Doctor med.

3) wie doch andere königliche Aerzte thaten; z. B. 17. Nov. 1534 disputavit de quodlibetaria M. Franciscus Myron, medicus Domini Delphini.

4) In den Facultätsacten heisst es z. B. 12. Nov. 1534 fuit vesperisatus M. Lodoicus le Tourneur. Celebratum est prandium in domo $\mathrm{m}$. nostri Vassetz, ubi post epulas mensaque remota tres formosi ac ingenta indole adulescentes una cum suo praeceptore suavissimo cantu et musicis dulce ferientibus instrumentis omnium assidentium aures atque animos incredibili gradam voluptate demulcerunt. 
durch Vorlesung einer Bittschrift, die er gegen Thibault selber angefertigt hatte. Die Facultät beschliesst sofort," das Gesuch beim Parlament einzureichen (17. Nov. 1534). Thibault ist persönlich in der Klage noch nicht genannt, sondern nur plusieurs gens ignares, empiriques et imposteurs, welche weder von der Medicin noch von der Astrologie irgend eine Kenntniss haben, und doch abergläubige und böchst thörichte Zukunftskalender herausgeben, und dadurch das Volk in allerlei religiöse Spaltungen und Ketzereien treiben (schismes et heresies). Das Parlament wird gebeten allen Druckern und Verkäufern solche Kalender und Zukunftsbüchlein zu verbieten, welche die Censur der Facultät nicht passirt haben (approuver ou reprouver). Als kläger gegen Thibault wird ein gewisser Brisard von der Facultät gedungen. Das Parlament antwortet, die Bittschrift solle dem Oberstaatsanwalt (procuratori generali Regis) übergeben werden (12. Dec. 1534). Der Oberstaatsanwalt verlangt Verweisung der Sache an den gewöhnlichen Gerichtshof.

Wenn man dem Tagault in eigener Sache glauben darf, so verbot der oberste Gerichtshof dem Leibarzt des Königs Jehan Thibault, fernerhin zu practisiren. Allein Thibault, dieser ,ganz ungelehrte, der Medicin zu grossem Schaden gereichende Mann", gab nicht nach. Schon hatte die Facultäts-Ausgabe (ungerechnet die 23 livres 15 sous 8 deniers für den gedungenen Kläger) um 538 livres 7 sous 3 deniers die Einnahme überschritten. "Dennoch, sagt Tagault, dankten mir die Magister für die Mühwaltungen und Kosten, die ich gegen diesen so niederträchtigen und dem ganzen Menschengeschlecht so feindseligen Menschen gehabt hätte (in prosequendo homine illo nefandissimo et naturae humanae hosti infestissimo), und baten mich, jeder einzeln und alle insgesammt, dass ich doch keine hosten sparen möchte, um diesen Jean Thibault weiter zu verfolgen."

Unter den neuen Kosten, welche die Fortführung des Prozesses "gegen den böchst einfältigen Empiriker" verursachte, verzeichnen die Register der Facultät z. B. für das Verbör von sechs Zeugen gegen Thibault 30 sous, für eine Schachtel feinen Zuckerwerks an die Adresse der Frau des Staatsanwalts (pour une boielle de dragée baillée à la femme de l'avocat du Roy) 15 sous, für „unsern Kläger" Brizard Erstattung der Unkosten 6 livres 15 sous, demselben als Facultätshonorar 10 livres, demselben an ausserordent- 
lichem Geschenk wegen seines Eifers bei Verfolgung des Thibault 4 livres 10 sous u. dgl. $\mathrm{m}$.

Den Erfolg hatte die Facultät unter Tagault's erstem Decanat, dass am 2. März 1535 ein Parlamentsbeschluss erzielt wurde, niemand in Paris dürfe practisiren, der nicht Pariser Doctor wäre oder wenjgstens Licentiat der Pariser Schule ${ }^{1}$ ). Und wäre er auch auf irgend einer andern Universität promovirt, so muss er doch, ehe er hier practisirt, vor vier Doctoren der Pariser Schule die Prüfung bestanden haben. Medicinische Schriften aber dürfen in Paris weder gedruckt noch verkauft werden, ehe sie nicht von drei aus der Facultät dazu erwählten Doctoren gebilligt worden sind. Das günstige Erkenntniss wurde in der Registratur der Facultät (in arca facultatis) aufbewahrt.

Das Princip war gegeben, und des Königs Leibarzt und Astrologe wurde von dem Parlament verurtheilt (21. März 1535). Aubery hatte den Thibault vertheidigt, Remon war für den 0berstaatsanwalt eingetreten, le Fèvre führte die Sache der Facultät ${ }^{2}$ ).

Am 17. Mai 1536. werden seitens der Facultät die drei Censoren committirt [Ludor. Braillon ${ }^{3}$ ), Michael de Monceau, Nicol. Guérin], welche in Gegenwart der königlichen Räthe De la Barde und Martin Fumée, des Empirikers Thibault Schriften, Le trésor de la Peste, de prognosticatione und Almanach prïfen sollten.

Man sollte nun meinen, mit der Verurtheilung durch das Parlament wäre die Sache abgethan gewesen. Doch nein, in dem königlich gewählten Leibarzt war des Königs Wahl gebrandmarkt worden. Wahrscheinlich hat daher Thibault an den König appellirt, und der König, nachdem Thibault sich dem Examen unter-

1) Ne aliquis deinceps praxin medicam exercere praesumat, in urbe Paris, et fourbiis ejusdem, nisi Doctor esset aut ad minus Licentiatus in schola Paris.

2) Den Extrait des Régistres du Parlement bringt du Boulay, VI, 264 flgd. cf. Staatsarchiv: Criminel $X, 8921$; contra Theobaldum empiricum.

3) Er war schon unter den Examinatoren (s. oben). Er ist 1534 medicus regius, ebenso Lodovicus Burgensis, Guill. Milet, Joh. Morelli, H le rongmus Varades; der Burgensis ist auch noch 19. März 1537 chevalier und premier médecin da Roy. $S$. Conseil $X, 1540$. Am 21.Jan. 1539 ist Me Jehan le Monete médecin ordinaire du Poy. S. Matinées $X$, 4907. - Im Jahre 1528 waren des Königs Aerzte Burgensis, Guil. Lecoq, Christofle de Forest, Jehan Goyreau. S. Heptaméron. Paris 1853. III. p. 276. 
worfen, die Sache an das Parlament zurückgewiesen. Jedenfalls finden wir nicht, dass Thibault die Prozess- und Prüfungskosten bezahlt hat, soudern diese füllen neve viertehalb Seiten der Facultätsacten aus ${ }^{1}$ ). Unter andern hatte eine Abhandlung über die Eitelkeit der geriehtlichen und prophetischen Astrologie (de vanitate astrologiae judiciariae et divinatrieis) dem Staatsanwalt Raimon und dem Facultätsadrocaten Lefèvre $z u$ inrer Instruction angefertigt und abgeschrieben werden müssen. Auch ersieht man aus dem Kostenverzeichniss, dass die königlichen Räthe Hennequin und le Rous den Thibault geprüft haben, ebenso Mag. Joh. Fernel (uni ex examinatoribus pd. Theobaldi).

Sehr geschickt war es, dass die Facullät verstanden hatte, des Thibault königliche Collegen gegen „den geschwätzigen Verleumder und albernen Herumtreiber" (contra sycophantam ac circulatorem nugacissimum, nebulonem ignoratissimum) za gewinnen. Kostete es der Facultät doch, wie es scheint, nur eine tüchtige Sendung jenes aromatischen Wein's, den man auch den hippokratischen nennt, und (für deren Damen?) einiges feine Zuckerwerk ${ }^{2}$ ). Dafür wussten die Leibärzte den König zu bewegen, Thibault förmlich fallen zu lassen in einer Cabinetsordre an den Chefpräsidenten des Parlaments ${ }^{3}$ ).

Der Decan hütet sich wohl, seine Niederlagen zu buchen. Die Facultätsacten erfahren nur von Siegen.

1) Darunter pro vino aromatico oblato primo praesidi, le jour du dimanche gras. - Viel Geld erhalten die Bedienten der Bäthe Hennequin, Fumée, Raimon, Lambert. Der letzte Posten ist Decano (also für Tagault selbst) pro laboribus inpensis in negociis facultatis administrandis 22 livres 10 sous: eine Belohnung, die Tagault beim Antritt seines ersten Decanats gleich eingeführt hatte.

2) Pro vino aromatico quod Hippocraticum vocant et tragematis oblatis medicis Regis, qui nobis impetraverant literas a Rege contra... Theobaldum directas ad primum Praesidem.

3) Der Sieg der Pariser Facultät flog durch das Land und machte andere medicinische Facultäten lïstern. Am 27. 0 ct. 1541 z. B. suppliciren die doctores fac. med. Pictav. beim Parlament von Poitiers: Il vous plaise faire inhtbitions et deffences, suyvant certain arrest de la court, donné contre Maistre Jehan Thibanlt à tous eulx disans médecins, de non pratiquer en ladite ville de Poitiers et sénéchaucé de Poictou .. que premlerement n'ayent esté oïz interroguez el experimentez par les maistres de la faculté. 
Indess die zwei Decanate Vassetz und die vier Tagault's genügen nicht, um Thibault zu vernichten. Unter dem Decanat des Anton Gallus (Lecoq) erscheint (1538-1539) ein neues Verzeichniss von Facultätsunkosten im Prozess gegen Thibault und gegen die astrologischen Prophetchen (adv. prognosticatorculos).

Der beste Beweis aber, dass Thibault seine Sache nicht verloren gab, ist der, dass im Prozess Tagault's gegen den spanischen jungen Astrologen, Stud. med. Michael Villanovanus - es ist Serveto - Jehan Thibault als Servet's adhérent et partie adverse de la dite Faculté de médecine erscheint ${ }^{1}$ ). Allerdings ist das Erkenntniss gegen seinen jungen spanischen Freund und Gesinnungsgenossen im Wesentlichen nur eine Bestätigung des gegen Thibault gefällten.

Gleich am 18. März 1538 wurde nun der Pedell der Facultät, Canto, ausgeschickt, um im Namen des Gerichtshofes auf Thibault's und der Andern Zukunftsbücher und Kalender Beschlag zu legen. Auch drei andern Empirikern und drei Aerztinnen (mulieribus empiricis practicantibus) sowie einigen Apothekern wurde das Erkenntniss mitgetheilt, das man gar gern mit Trompetenschall (sono tubae) durch die Stadt hätte verkünden lassen: aber der oberste Gerichtshof ging nicht darauf ein.

Johann Thibault war aus der Zunft der Aerzte ausgestossen (supremo senatus judicio a medicorum ordine remotus). Dennoch erscheint er am 3. Februar 1538 vor Gericht als conseiller et médecin ordinaire du Roy, gewinnt einen Prozess gegen Guentin Thire ${ }^{2}$ ), und giebt wieder neue Almanach's heraus ${ }^{3}$ ), die nicht blos von der Facultät nicht gebilligt worden sind, sondern sogar zwei "ketzerische" Artikel enthielten (sacrae nostrae fidei adversantes). Dafür behandelt die Facultät den königlichen Leibarzt als einen unsaubern Idioten (vir impurus et indoctus), seine Bücher als höchst lächerlich und seine Freunde als die zur Ausrottung des christlichen Glanbens frevelhaft Verbündeten. Und die andern Facultäten pflichten bei.

1) S. Mosheim: Anderweit. Versuch. 401.

2) Subrogue au lieu et droict de Philippes de Silly et ledit Silly appellants du prérost de Paris: Matinées X, 4907 (Archives génér. de la France). Die Benutzung dieser Archive wurde mir im Winter 1858-1859 mit grösster Liberalität gestattet.

a) Prognostications pour les années 1539-1544. 
Der Niederländer Thibault ist unterdessen in Paris ein doppelt bekanter Mann geworden, durch sein enormes practisches Geschick und durch sein seltenes Ungemach. Bei Chrét. Wechel in Paris giebt er les tables du soleil et de la lune heraus (1534-1540), ebenfalls zu Paris eine Table de la dignité des Planètes (c. 1540), bej Denys Gaignot in le Mans die Grande et merveilleuse prophétie (1545-1556) u. s. f. Diese Bücher Thibault's habe ich nie zu Gesicht bekommen ${ }^{1}$ ), bin aber weit entfernt zu behaupten, dass kein Exemplar mehr davon existire. Auch die von keiner Seite verbotenen Kalender und Sternbüchlein ${ }^{2}$ ) waren so schnell vergriffen, wie heute die Flugblätter und Extrablätter unserer Zeitungen ${ }^{3}$ ). Kein Urtheil möchte so irrig sein, als wollte man behaupten, was im Buchhandel heute selten ist, ist im Buchhandel nie verbreitet gewesen ${ }^{4}$ ): gerade das Gegentheil trifft oft zu.

Es ist natürlich, dass bei dem aufreibenden Kampf, welcher der Facultät sehr viel Geld kostete, der protestantisch angebauchte Niederländer damit endete, sich überall Feinde zu machen. Schlimwer aber war der entfesselte Spott. Unmöglich kann ein Prophet immer das Richtige voranssagen. Auch Maître Thibault hatte endlich in Paris sich lächerlich gemacht, und die zeitgenössischen Dichter erhoben seine Schwächen zur Unsterblichkeit.

Mellin de St. Gelais, ein bei den Herren und Damen des Hofes damals sehr beliebter leichtfertiger Abt $\left.\left({ }^{\prime} 1558\right)^{5}\right)$ liess folgendes Gedicht umlaufen:

Maistre Jean Thibaut va jurant

Qu'il n'est ni fol ni esventé

Et encores moins ignorant,

El qu'il a tout seul inventé

L'escrit qu'un autre s'est vanté

1) De la Croix du Maine cltirt sie im Artikel Thibault.

2) z. B. Carion's.

3) Die Kalender enthielten regelmässig die politischen Daten des vergangenen Jahres und sind, seit Ende des XV. Jahrhunderts, „das erste Muster der Zeitungsliteratur". Graesse, Literärgeschichte des XVI. Jahrb. III, 32.

4) wie die physiologischen Gegner Servet's zu schliessen lieben, weil heute von Servet's Restifutio nur 2 echte vollständige Exemplare vorhanden sind, könne die Restitutio nie nach Italien, Spanien a. s. w. verbreitet worden sein.

5) Démogeot, Hist. de la Litérature franç. Paris 1862. 326. 
D'avoir faict du tourner des cieux.

Maistre Jean Thibaut faites mieux,

Donnez luy le livre et l'estoffe,

Et l'on tiendra vostre envieux

Pour un très mauvais philosophe ${ }^{1}$ ).

Ein anderes geht tiefer in die Hofgeheimnisse ein:

Jehan Thibault entre ses amis

Se lamente en toute saison

Du Roy qui luy avoit promis

De luy donner une maison;

Mais le fol se plaint sans raison;

Car le Roy a bien peu savoir

Que Jehan Thibault nul ne va voir

Pour rire, ainsi que nous faisons,

A qui pour quelque argent avoir

Il n'érige douze maisons.

Auch über Thibaut's Gutmüthigkeit erlustigt sich der höfische Abbé:

Vous faites bien, maistre Thibault,

De garder ce que vous avez,

Nulle chose non plus ne vaut

Qu'on la prise ainsi que savez.

Prester vos extraits ne devez

Pour bien qu'en puissiez acquérir.

Mais moy qui n'ay rien que chérir,

Je mets tous mes papiers en voye,

Tant que mesme sans rien quérir

Ces vers icy je rous envoye ${ }^{2}$ ).

Von wem das Gedicht stammt, das handschriftlich dem De la Monnoye mitgetheilt wurde aus dem Jahre 1544, lässt sich nicht mehr sagen. Es lautet:

As-tu ouĩ parler du veau

Naguères né avec deux têtes?

C'est signe que les grosses bêtes

Porteront pour deux cornes quatre,

1) Oeuvres de Mellin de St. Gelais. Paris, ed. 1719. p. 135.

2) 1. 1. p. 227 . 
De fait Jean Thibaut le folâtre

L'a tout ainsi pronostiqué.

De la Croix du Maine ${ }^{x}$ ) setzt Jean Thibault's Tod um 1544. Ich bin nicht gewillt, zu leugnen, dass der originelle Leibarzt und Leibastrologe Franz I., Michael Servet's Freund, vieles an sich gehabt haben mag, was ihn lächerlich machte. Bedenkt man aber, angesichts von Thibault's Aufopferung für die armen Pestkranken, dass „bei Epidemieen die Beschäftigung des Arztes in umgekehrtem Verhältniss zu seiner Einnahme steht ${ }^{2}$ )“, und aass, als Thibault nach Paris kam, das Parlament die Facultät zwingen ${ }^{3}$ ) musste, die Pestkranken zu besuchen ${ }^{4}$ ), die Facultät aber den erwählten vier Pestärzten (médecins Docteurs régens de qualité tant en theorica que practica) versprechen musste, jedem für das laufende Pestjahr ${ }^{5}$ ) 300 livres parisiens auszuzahlen, wogegen er sich, bei harter Strafe, aller übrigen Praxis zu enthalten babe (1532): so wird man doch dem warmen Freund der Armen, dem muthigen Vertreter des Experiments ${ }^{6}$ ), dem Vf. des trésor de la peste für sein uneigennütziges, unerschrockenes, freisinniges Rettungswerk im Namen der leidenden Menschheit noch nach seinem Tode Dank wissen.

1) Er nennt ihn médecin ordinaire de Louis XII.

2) Ed. Wolff, Ueber den Stand des Arztes. 1862. S. 42.

3) Panition corporelle, privation de leurs offices et amende arbitraire werden angedroht.

4) Pour visiter et médicamenter les malades de la peste en ceste ville et faulxbourgs dicelle.

5) 1530-1540 kommen in den Parlamentsacten von Paris immer wieder vor les salaires pour les médecins, chirurglens, barbiers et autres ordonnez pour les pestiferez de la ville de Paris.

6) Paracelsus III, 301: Die Kranken sollen des Arztes Bücher sein. Lesen hat nie einen Arzt gemacht, sondern die Practik. Vgl. Lessing, Leben des Paracelsus. S. 61 flgd. 